\title{
Isoscaling in dissipative projectile breakup
}

\author{
H. Singh ${ }^{1}$, M.J. Quinlan ${ }^{1}$, J. TÕKE ${ }^{1}$, I. PAWElczaK ${ }^{1}$, E. Henry ${ }^{1}$, \\ W.U. Schröner ${ }^{1}$, F. Amorini ${ }^{2}$, A. Anzalone ${ }^{2}$, C. Maiolino ${ }^{2}$,

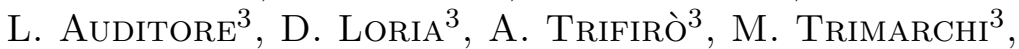 \\ G. Cardella ${ }^{4}$, E. De Filippo ${ }^{4}$, A. Pagano ${ }^{4}, \mathrm{M}_{\text {. Papa }}{ }^{4}$,
} S. Pirrone ${ }^{4}$, G. Verde ${ }^{4}$, M.B. ChatterJee ${ }^{5}$, S. Cavallaro ${ }^{2,6}$, E. Geraci ${ }^{4,6}$, S. Lo Nigro ${ }^{6}$, G. Politi ${ }^{4,6}$, F. Porto ${ }^{2,6}$, F. Rizzo $^{2,6}$, P. Russotto ${ }^{2,6}$, A. Grzeszczuk ${ }^{7}$, P. Guazzoni ${ }^{8}$, L. Zetta $^{8}$, E. La Guidara ${ }^{4,9}$, G. Lanzalone ${ }^{10}$, M. Vigilante ${ }^{11}$

${ }^{1}$ Departments of Chemistry and Physics, University of Rochester, Rochester NY, USA

${ }^{2}$ INFN Laboratori Nazionali del Sud, Catania, Italy

${ }^{3}$ INFN-Gr. Coll. Messina and Dip. di Fisica Università, Messina, Italy

${ }^{4}$ INFN Sez. di Catania, Italy

5 Saha Institute for Nuclear Physics, Kolkata, India

6 Dip. di Fisica e Astronomia, Università di Catania, Italy

7 Institute of Physics, University of Silesia, Katowice, Poland

${ }^{8}$ INFN Sez. di Milano and Dip. di Fisica Università di Milano, Italy

9 CSFNSM, Catania, Italy

10 INFN LNS, Catania and Università Kore Enna, Italy

11 INFN Sez. di Napoli and Dip. di Fisica Università, Napoli, Italy

\begin{abstract}
Dynamical breakup of projectile-like fragments (PLF) following dissipative reactions of ${ }^{48} \mathrm{Ca}$ projectiles with ${ }^{112} \mathrm{Sn}$ and ${ }^{124} \mathrm{Sn}$ is shown to exhibit "isoscaling" regularities that can be understood in terms of phase space governed by ground state masses. Ambiguities in isoscaling parameters obscure information on nuclear symmetry energy at subnormal densities.
\end{abstract}




\section{Introduction}

The CECIL collaboration has studied multi-particle correlations in ${ }^{48} \mathrm{Ca}+{ }^{112} \mathrm{Sn}$ and ${ }^{48} \mathrm{Ca}+{ }^{124} \mathrm{Sn}$ reactions at $\mathrm{E} / \mathrm{A}=45 \mathrm{MeV}$ using the CHIMERA array [1]. Experimental objective was to explore regularities in the isotopic distributions of products emitted following dissipative interactions [2] of $\left({ }^{48} \mathrm{Ca}\right)$ with targets of different $\mathrm{A} / \mathrm{Z}$, in relation to the nuclear symmetry energy $[3,4]$ at subnormal matter densities that could be accessible $[5,6]$. Attention was paid to the possible contribution of non-equilibrium effects [7] and the degree of equilibration reached by the systems. Earlier work [8] had demonstrated the role of pre-equilibrium emission altering nuclear identities and limiting excitation.

\section{Experimental procedures}

Isotopically enriched, self-supporting targets of ${ }^{124} \mathrm{Sn}$ and ${ }^{112} \mathrm{Sn}$ with thicknesses of $689 \mu \mathrm{g} / \mathrm{cm}^{2}$ and $627 \mu \mathrm{g} / \mathrm{cm}^{2}$, respectively, placed in the center of CHIMERA, were bombarded with pulsed, $\mathrm{E} / \mathrm{A}=45-\mathrm{MeV},{ }^{48} \mathrm{Ca}$ beams from the LNS K800 cyclotron. ${ }^{12} \mathrm{C}$ and ${ }^{16} \mathrm{O}$ beams from the LNS MP Tandem were used for detector calibration, along with elastically scattered ${ }^{48} \mathrm{Ca}$ projectiles. Reaction products were characterized by atomic number Z, mass number A, energy and emission angles, utilizing time-of-flight (TOF), energy and light output information provided by the $S i-C s I$ telescopes.

The present study focusses on data collected in the forward angular region $\left(6^{\circ} \leq \theta \leq 20^{\circ}\right)$, comprising projectile-like fragments (PLF) and their decay products, much of it in form of correlated pairs of an IMF $(3 \leq \mathrm{Z} \leq 5)$ and a corresponding heavier PLF remnant. Experimental details are provided elsewhere [9].

\section{Reaction mechanism}

An overview over the reaction scenario is provided in Fig. 1 displaying yields for the reaction ${ }^{48} \mathrm{Ca}+{ }^{112} \mathrm{Sn}$ in form of Wilczyński-type contour diagrams of reconstructed PLF kinetic energy ( $\mathrm{T}_{P L F}$, top), or velocity $\left(|\mathrm{v}|_{P L F}\right.$, bottom), vs. lab angle.

Obviously, average yields evolve with PLF laboratory angle and kinetic energy as expected [2] for a dissipative reaction. Calculations with dissipative reaction code $C L A T$ [10] (Fig. 1a), symbols and curves) agree quantitatively with the data. Open symbols indicate primary yields, solid ones 


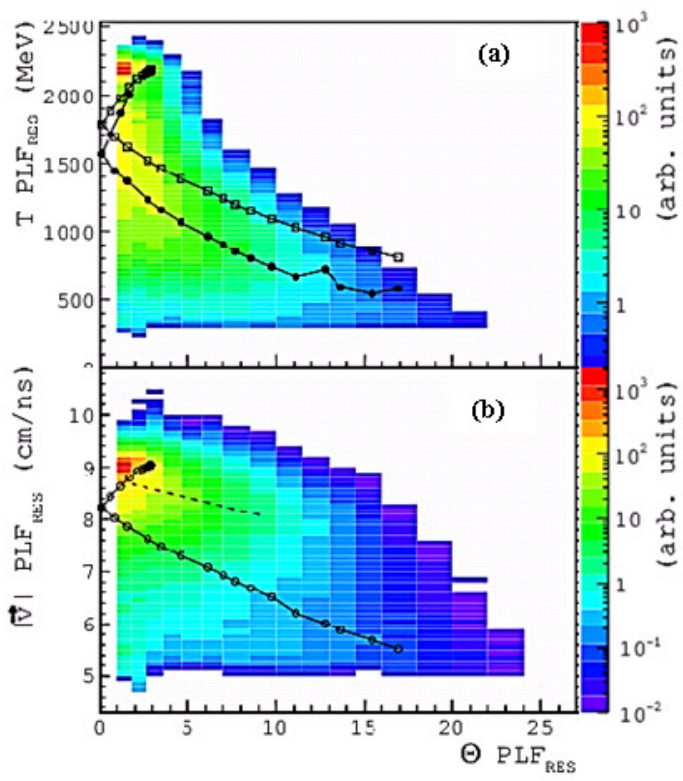

Figure 1: Experimental Wilczyński contour diagrams for the reaction ${ }^{48} \mathrm{Ca}+{ }^{124} \mathrm{Sn}$ at $\mathrm{E} / \mathrm{A}=45 \mathrm{MeV}$. Top: PLF energy vs. angle, bottom: PLF velocity vs. angle. Symbols and curves represent mean predictions by the nucleon exchange model (CLAT). See text.

account for evaporation calculated with GEMINI [11]. However, plotting yields (Fig. 1b) vs. PLF velocity instead of energy, significant discrepancies between theory and data appear. Less velocity damping occurs than predicted, indicating a fortuitous agreement between theory and kinetic-energy data in Fig. 1a.

The above inconsistencies are attributed to dynamical breakup of the PLF following dissipative interactions, instead of statistical decay assumed in the CLAT/GEMINI simulations. The PLF breakup mechanism is not predicted by microscopic $Q M D$ simulations [12], either, but is supported by several pieces of experimental evidence: Neither heavy nor light PLF remnants exhibit random Galilei invariant cross section patterns expected for statistical decay but show strong forward/backward asymmetries of both breakup cross section and mass asymmetry, as well as large relative velocities of the PLF breakup fragments [9]. Dynamical PLF breakup resembling ternary fission $[13,14]$ is well known at Fermi energies [15], especially for cluster nuclei. Potential energy surfaces evaluated for the present systems support the observed evolution of the reaction mechanism with relative angular momentum. 


\section{Isoscaling in PLF breakup}

\subsection{General considerations}

Since the symmetry energy at subnormal mass densities is currently of high interest, the investigation focussed on nuclear clusters which, unlike nucleons, can be emitted statistically only from hot, diluted nuclei $[5,6]$. Therefore, early work [16] on cluster emission in low-energy reactions did not provide new insights into the density dependence of the symmetry energy. To substantially dilute matter requires high excitation which, unfortunately, associates with non-equilibrium emission [7].

To reduce susceptibility to systematic uncertainties, previous work [17-21] considers ratios $R_{12}$ of cluster $\left(N_{c}, Z_{c}\right)$ yields from different parent nuclei $(i=1,2)$ at similar excitations. Since the nuclear binding energy depends quadratically on neutron and proton numbers, statistical yields are approximately Gaussians. Consequently, yield ratios depend approximately exponentially on $N_{c}$ and $Z_{c}$ :

$$
R_{12}\left(N_{c}, Z_{c}\right)=Y_{2}\left(N_{c}, Z_{c}\right) / Y_{1}\left(N_{c}, Z_{c}\right) \propto \exp \left\{\alpha N_{c}+\beta Z_{c}\right\}
$$

Here, isoscaling parameters $\alpha$ and $\beta$ reflect principal curvatures of the $\beta$ stable valley for the two emitters $(i=1,2)$ at the effective temperature. For systems at constant temperature $\mathrm{T}$, the following relations have been suggested $[17,18]$ between isoscaling parameters and symmetry energy coefficient $C_{\text {sym }}$ :

$$
\beta=C_{\text {sym }} \Delta(N, A) / T ; \quad \alpha=C_{\text {sym }} \Delta(Z, A) / T ;
$$

with the neutron or proton excess difference functions $\Delta$ defined as

$$
\Delta(X, A)=4\left[(X / A)_{1}^{2}-(X / A)_{2}^{2}\right]
$$

\subsection{Experiment results and discussion}

In the following, measured cluster isotope yields from $P L F^{*}$ breakup are plotted as ratios vs. cluster neutron or proton numbers $(N, Z)$ in the form

$$
\operatorname{Ratio}(N, Z)=Y_{N, Z}\left({ }^{48} \mathrm{Ca}+{ }^{124} \mathrm{Sn}\right) / Y_{N, Z}\left({ }^{48} \mathrm{Ca}+{ }^{112} \mathrm{Sn}\right)
$$

Measured Li, Be, B, C and $\mathrm{N}$ isotope ratios shown in Fig. 2 vs. cluster neutron number $\mathrm{N}$, demonstrating isoscaling, where isotopic ratios trace parallel logarithmic straight lines. Isotone ratios show corresponding behavior when 


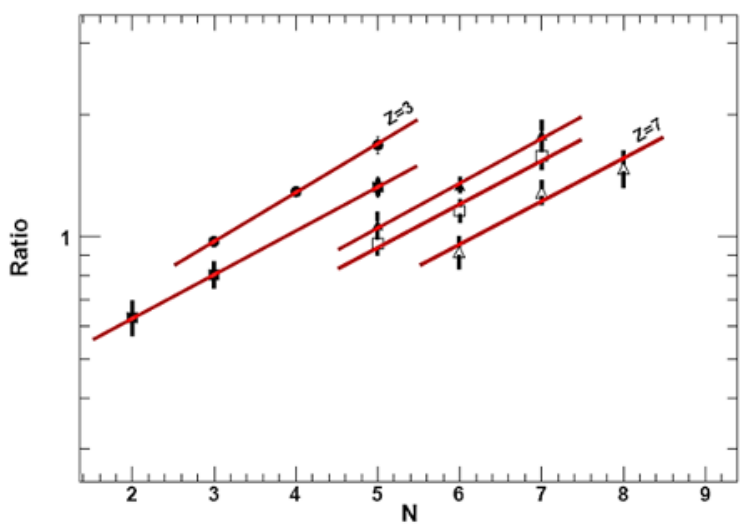

Figure 2: Isoscaling plot for Li, Be, B, C, and N clusters from PLF breakup.

plotted vs. cluster- $Z$ value. These observations contrast with other studies of projectile fragmentation $[22,23]$ showing isoscaling effects only after $P L F$ reconstruction.

In further data reduction, average ("global") isoscaling parameters of $\bar{\alpha}=(0.23 \pm 0.02)$ and $\bar{\beta}=(-0.12 \pm 0.01)$ were obtained from simultaneous fits to all isotope or isotone ratios. The errors contain systematic uncertainties evaluated from variations of constraints imposed on data selection. It is interesting that the present parameters are by factors of 3 to 4 smaller than reported for other projectile fragmentation studies [17-24].

The further analysis varied $P L F_{i}^{*}$ atomic and mass numbers, with differences $\Delta B=B_{2}-B_{1}$ in energy cost for emission from $P L F_{1}^{*}$ and $P L F_{2}^{*}$ evaluated for all clusters from ground state masses, until the entire data set was on average well reproduced by the exponential

$$
\begin{aligned}
R_{21}(N, Z) & =Y_{N, Z}\left({ }^{48} C a+{ }^{124} S n\right) / Y_{N, Z}\left({ }^{48} C a+{ }^{112} S n\right) \\
& \approx \exp \left\{\frac{\left[B_{2}(N, Z)-B_{1}(N, Z)\right]}{T_{e f f}}\right\}=\exp \left(\frac{\Delta B}{T_{\text {eff }}}\right)
\end{aligned}
$$

Identical effective temperatures $T_{e f f}$ were taken for both emitters. Experimental energy spectra suggest $3 \mathrm{MeV} \leq T_{\text {eff }} \leq 4 \mathrm{MeV}$ as acceptable range.

Results are depicted in Fig. 3 for experimental yield ratios vs. cluster ground state plotted vs. binding energy differences $\Delta B$ for breakup pair $P L F_{1}^{*}=\left(Z_{1}=20, A_{1}=49\right)$ and $P L F_{2}^{*}=\left(Z_{2}=18, A_{2}=43\right)$. The line drawn through the data corresponds to an effective temperature $T=$ $(2.6 \pm 0.3) \mathrm{MeV}$. An equally good fit is obtained using the pair $P L F_{1}^{*}=$ 


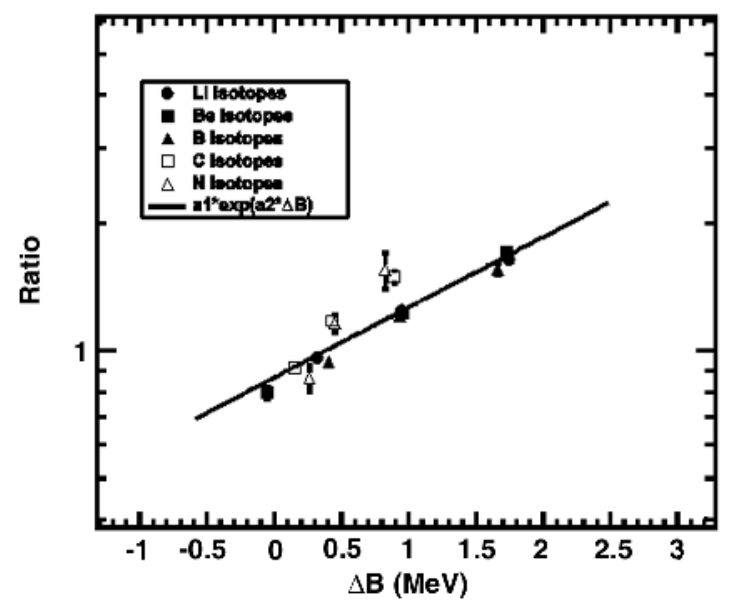

Figure 3: Scaling of experimental cluster yield ratios for ${ }^{48} \mathrm{Ca}+{ }^{124,112} \mathrm{Sn}$ reactions at $\mathrm{E} / \mathrm{A}=45 \mathrm{MeV}$ based on ground-state binding energy differences (cf. Eq. 5).

$\left(Z_{1}=25, A_{1}=48\right)$ and $P L F_{2}^{*}=\left(Z_{2}=26, A_{2}=49\right)$. However, this latter case corresponding to significant proton pickup and a high value $T_{\text {eff }}=$ $(5.5 \pm 0.3) \mathrm{MeV}$ is deemed highly unlikely, based on driving potentials and similar ${ }^{40,48} \mathrm{Ca}+{ }^{112} \mathrm{Sn}$ experiments [25].

Discrepancies between present and literature isoscaling parameters pose the question whether or not scaling with ground state binding energies (cf.

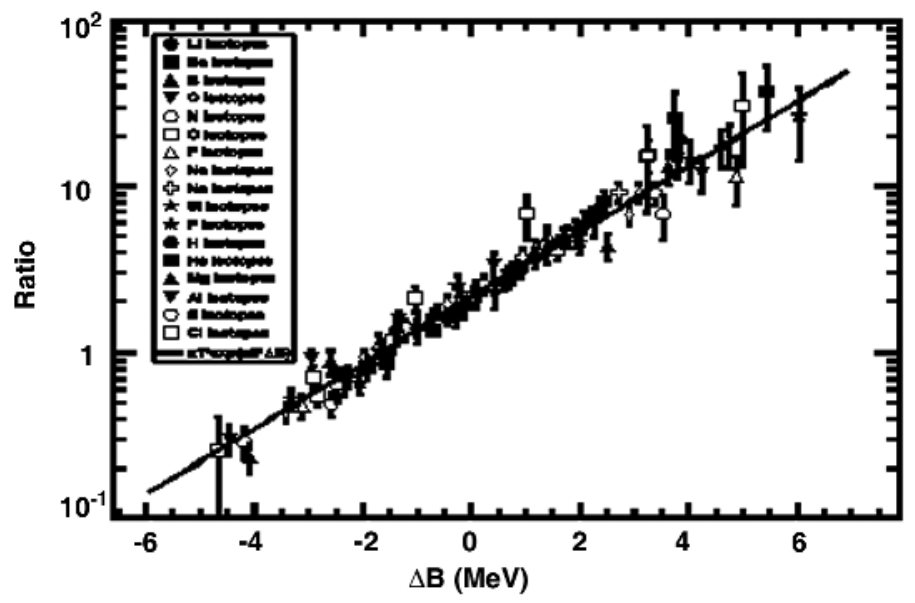

Figure 4: Scaling of experimental yield ratios with binding energy differences for ${ }^{86,78} \mathrm{Kr}+{ }^{64,58} \mathrm{Ni}$ reactions at $\mathrm{E} / \mathrm{A}=35 \mathrm{MeV}$. Data imported from [22]. 
Fig. 3) is specific to the present reactions. The question is answered by results (cf. Fig. 4) of a similar analysis of data [22] for the reactions ${ }^{86} \mathrm{Kr}$ $+{ }^{64} \mathrm{Ni}$ and ${ }^{78} \mathrm{Kr}+{ }^{58} \mathrm{Ni}$ at $\mathrm{E} / \mathrm{A}=35 \mathrm{MeV}$.

For the latter reactions, search for a $P L F^{*}$ pair whose ground state binding energy patterns represent experimental yield ratios yielded $P L F_{1}^{*}=$ $\left(Z_{1}=40, A_{1}=89\right)$ and $P L F_{2}^{*}=\left(Z_{2}=39, A_{2}=84\right)$ for a reasonable temperature of $T=(2.2 \pm 0.2) \mathrm{MeV}$. As shown in Fig. 4, experimental data [22] are well fit with this parameterization. Data from the present experiment included in this figure merge well with the ${ }^{78,86} \mathrm{Kr}+{ }^{58,64} \mathrm{Ni}$ data demonstrating a remarkable agreement of diverse data sets with a groundstate binding-energy systematics.

\section{Conclusions}

In summary, experimental data are presented for a dynamical breakup process of a fairly light projectile $(\mathrm{Ca})$ following a dissipative primary reaction. The breakup produces intermediate-mass clusters exhibiting isoscaling consistent with populations according to ground-state $Q$ values and symmetry energies at normal density. Therefore, such reaction data do not provide unambiguous symmetry energies at subnormal matter densities. Ambiguities can be reduced by precise knowledge about underlying reaction mechanisms involving simpler composite systems produced close to equilibrium.

\section{Acknowledgments}

Work supported by the US Department of Energy Grant No. DE-FG0288ER40414. Authors express their gratitude to the LNS for hospitality and excellent beams.

\section{References}

[1] A. Pagano et al., Nucl. Phys. A 734, 504 (2004).

[2] W.U. Schröder and R.J. Huizenga, Treatise on Heavy Ion Science, edited by D.A. Bromley (Plenum Press, New York, 1984).

[3] J.P. Blaizot, Phys. Reports 64, 171 (1980).

[4] B.A. Li, L.W. Chen, C.M. Ko, Phys. Reports 464, 113 (2008) and references. 
[5] J. Tõke and W.U. Schröder, Phys. Rev. Letters 82, 5008 (1999).

[6] J. Tõke, J. Lu and W.U. Schröder, Phys. Rev. C 67, 034609 (2003).

[7] E. Gadioli and P.E. Hodgson, Pre-Equilibrium Nuclear Reactions, Clarendon Press, Oxford (1992).

[8] W.U. Schröder and J. Tõke, Nucl. Phys. A 681, 418c (2001).

[9] M.J. Quinlan, PhD Thesis, University of Rochester, 2011, and to be published.

[10] W.U. Schröder et al., Proc. Int. Symp. Nucl. Fission and Heavy-IonInduced Reactions, Harwood Academic Press, New York, Nucl. Sci. Res. Conf. Ser. Vol. 11, 255 (1987).

[11] R.J. Charity et al., Nucl. Phys. A. 483, 371 (1988).

[12] J. Lukasik and Z. Majka, Acta Phys. Pol. 24, 1959 (1993).

[13] I. Halpern, Annu. Rev. Nucl. Sci. 21, 245 (1971).

[14] J.P. Lestone, Phys. Rev. C 70, 021601(R) (2004).

[15] H. Fuchs and K. Möhring, Rep. Prog. Phys. 57, 231 (1994).

[16] V.V. Volkov, Phys. Rep. 44, 93 (1978) and references.

[17] M. B. Tsang et al., Phys. Rev. Lett. 86, 5023 (2001).

[18] A.S. Botvina et al., Phys. Rev. C. 65, 044610 (2002).

[19] J. Brzychczyk et al., Phys. Rev. C. 47, 1553 (1993).

[20] Z. Chen et al., Phys. Rev. C. 81, 064613 (2010).

[21] A.B. McIntosh et al., Phys. Rev. C 81, 034603 (2010).

[22] S. Wuenschel et al., Phys. Rev. C. 79, 061602(R) (2009).

[23] S. Galanopoulos et al., Nucl. Phys. A. 837, 145 (2010).

[24] D. V. Shetty et al., Phys. Rev. C. 71, 024602 (2005).

[25] W.U. Schröder et al., Isospin Phys. Heavy-Ion Coll. Interm. Energies, p.303, (Li,Schröder,Eds), Nova Science Publ., Huntington, New York, 2001. 